\title{
Rassegna
}

\section{Nuove linee guida ISPD per il trattamento della peritonite in dialisi peritoneale: cosa c'è di nuovo?}

\author{
P. Lisi, R. Corciulo, R. Russo \\ U.O. di Nefrologia, Azienda Ospedaliero-Universitaria Consorziale, Policlinico di Bari
}

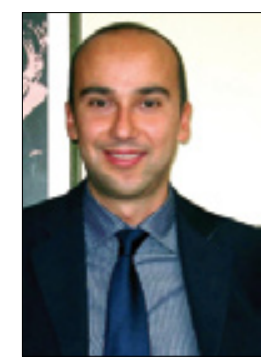

Piero Lisi
La peritonite rappresenta tuttora la principale complicanza legata alla dialisi peritoneale, ed è responsabile di una significativa morbilità e in alcuni casi mortalità. Infatti, circa il 18\% della mortalità da cause infettive è correlata alle peritoniti. Inoltre, determinando un'alterazione strutturale e funzionale della membrana peritoneale, essa è causa d'interruzione della metodica e passaggio al trattamento emodialitico (1-6). L’incidenza di peritonite si è ridotta negli ultimi 15 anni da circa un episodio ogni 9 mesi per paziente, a uno ogni 24 mesi. Questo è coinciso con il passaggio dal sistema a sacca singola al sistema a doppia sacca (twin bag) (7). Le principali vie di contaminazione peritoneale sono quella endoluminale, durante le manovre di connessione, la periluminale secondaria all'infezione dell'emergenza cutanea, e quella endogena favorita da condizioni che alterano la permeabilità intestinale (diarrea e diverticolite). Nei primi due casi, i germi più frequenti sono lo Stafilococcus aureus e lo Stafilococcus epidermidis; nel secondo caso i gram-negativi. Le infezioni fungine possono comparire dopo cicli prolungati di antibiotici (7).

Dal 1983, l'International Society of Peritoneal Dialysis (ISPD) ha elaborato delle linee guida sulla prevenzione, la diagnosi e il trattamento delle infezioni correlate alla dialisi peritoneale. Queste si basano su studi randomizzati controllati e, in mancanza di evidenza, su opinioni maturate sulla base di sufficiente esperienza. Le linee guida sono state revisionate e aggiornate nel 1989, 1993, 1996, 2000, e 2005. Nelle precedenti era presente una parte riguardante la prevenzione; in quelle del 2010 questa parte è stata eliminata, ed è stata focalizzata l'attenzione su diagnosi e trattamento delle peritoniti (8).

\section{Presentazione clinica delle peritoniti e terapia empirica}

Tutti i pazienti in dialisi peritoneale che presentano un liquido di drenaggio torbido, devono essere considerati affetti da peritonite, fino a prova contraria. Di solito il paziente lamenta anche dolori addominali che possono avere intensità e durata variabili. La conferma è data dagli esami colturali, con metodiche standardizzate che riducono la percentuale di falsi negativi (8).

Benché già raccomandato nelle precedenti linee guida, oggi si rafforza il concetto dell'inizio di una terapia antibiotica empirica, eventualmente modificata secondo antibiogramma, al fine di ridurre al minimo l’insorgenza di complicanze.

La terapia dovrebbe essere ad ampio spettro, contro gram-positivi e gram-negativi, per via di somministrazione intraperitoneale (IP). Gli studi hanno dimostrato l'efficacia, come prima scelta, della vancomicina o di una cefalosporina verso i gram-positivi, e delle cefalosporine di terza generazione o degli aminoglicosidi verso i gramnegativi. Comunque è importante, per ogni Centro, attuare protocolli che tengano conto della storia locale della sensibilità dei ceppi isolati, monitorando l'insorgenza di resistenze e considerando le eventuali tolleranze farmacologiche (Tab. I).

Oltre agli schemi terapeutici alternativi già conosciuti (cefepime o ceftazidime per i gram-negativi, monoterapia con imipenem/cilastatina o con cefepime, chinolonici in alternativa agli aminoglicosidi), dal 2005 a oggi sono stati studiati diversi tipi di antibiotici: l'associazione cefazolina e netilmicina IP è risultata efficace come trattamento empirico quanto la combinazione di cefazolina e ceftazidime IP (9); inoltre, entrambe non hanno avuto influenza sulla funzione renale residua. Ancora, sono 
TABELLA I - TRATTAMENTO INIZIALE DELLA PERITONITE

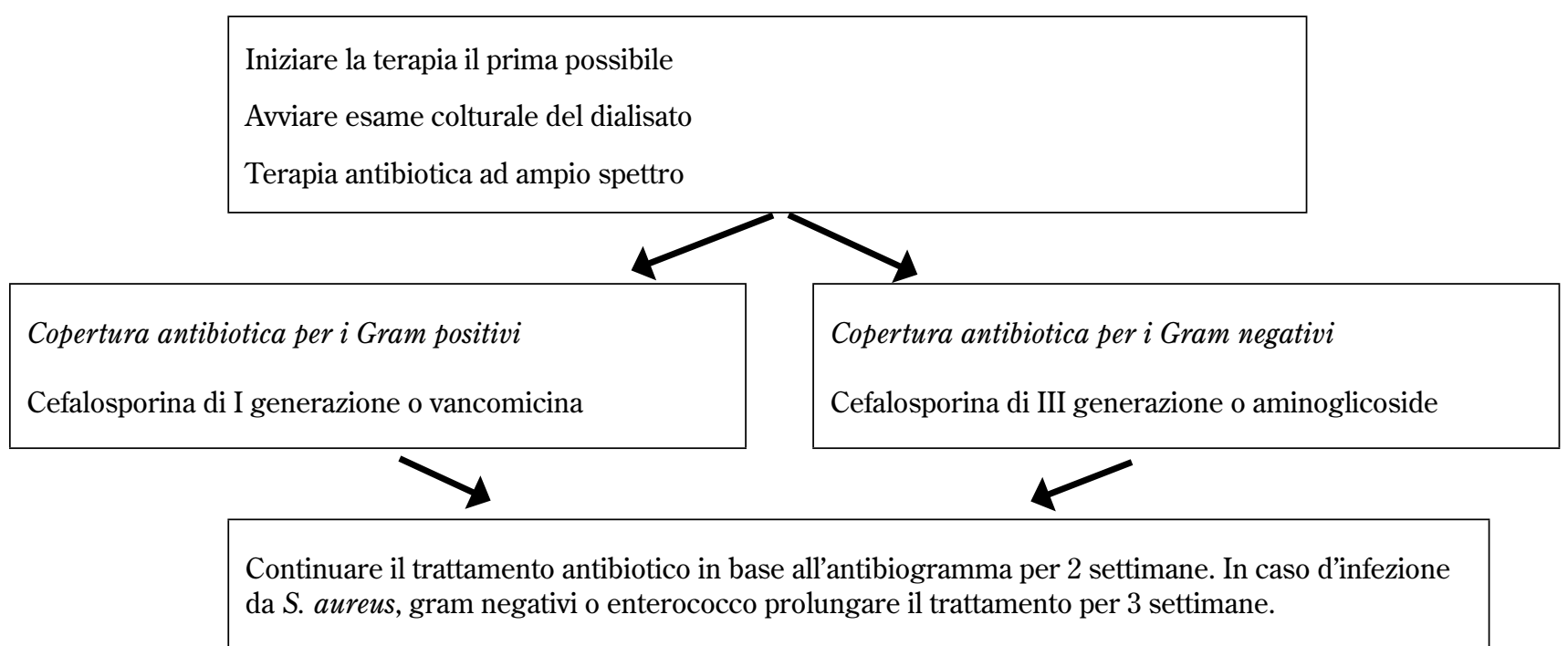

stati testati con successo vancomicina in associazione alla ciprofloxacina per via sistemica (10), o ciprofloxacina e cefazolina (11). Un altro schema, meropenem e tobramicina, seguito da meropenem più vancomicina dovrebbe essere utilizzato per i casi con storia di multiresistenza (12).

La via di somministrazione IP in CAPD, rispetto a quella endovenosa (e.v.), ha dimostrato di essere più efficace per il trattamento delle peritoniti (13), per la più alta concentrazione locale che il farmaco raggiunge, oltre che più vantaggiosa per l'uso domiciliare in pazienti opportunamente addestrati. Vancomicina, aminoglicosidi e cefalosporina possono essere mescolate nella stessa sacca di dialisi, utilizzando siringhe separate. Gli aminoglicosidi sono incompatibili con le penicilline.

La somministrazione può essere fatta in ogni scambio (continua) o una volta al giorno (intermittente); in quest'ultimo caso, la soluzione di dialisi deve stazionare per almeno 6 ore (8). Per la gran parte degli antibiotici esistono dati sulla somministrazione continua o intermittente in CAPD, eccetto che per le penicilline, la ciprofloxacina, l'aztreonam e la daptomicina, per i quali non si conosce il dosaggio ottimale nel caso di somministrazione intermittente. Non è dimostrata un'efficacia superiore, nella somministrazione continua, per le cefalosporine di prima generazione. Ci sono ancora pochi studi sulla dose di antibiotico richiesta dai pazienti in APD con somministrazione intermittente.

A proposito del dosaggio, recenti studi hanno confermato che le dosi intraperitoneali di vancomicina utilizzate nei pazienti in CAPD e in APD, determinano un'adeguata concentrazione sierica nella maggior parte dei pazienti.
Al contrario, le dosi raccomandate di gentamicina determinano livelli sierici elevati del farmaco nella metà dei pazienti considerati. Inoltre, incrementando il dosaggio dei due suddetti antibiotici, non si ottiene un beneficio superiore in termini di efficacia (14).

\section{Gestione delle peritoniti dopo esame colturale}

Conosciuto l'agente responsabile, la terapia antibiotica deve essere modificata secondo antibiogramma, dosando adeguatamente gli antibiotici ad escrezione renale nei pazienti che hanno una funzione renale residua $(>5$ $\left.\mathrm{ml} / \mathrm{min} / 1.73 \mathrm{~m}^{2}\right)(8)$.

Dopo 48 ore circa dallinizio della terapia, la maggior parte dei pazienti dovrebbe presentare un miglioramento del quadro clinico. Il liquido di drenaggio dovrebbe essere esaminato quotidianamente per verificarne il graduale ritorno alla limpidezza. In caso contrario, sarebbe opportuno ripetere gli esami colturali e la conta cellulare. Una conta cellulare del dialisato peritoneale $\geq \operatorname{di} 1090 / \mathrm{mm}^{3}$ al terzo giorno di terapia è un marcatore prognostico indipendente di fallimento della terapia in atto (15).

Se dopo circa cinque giorni di trattamento con antibiotico adeguato non avviene alcun miglioramento, si tratta di peritonite refrattaria. In questo caso è indicata la rimozione del catetere peritoneale, onde evitare morbidità $\mathrm{e}$ mortalità, per preservare la membrana peritoneale $(3$, $15,16)$.

Uno studio del 2009 (17) mostra che gli episodi di peritoniti recidivanti e ricorrenti sono causati da tipi diversi di batteri e probabilmente rappresentano due distinte 
entità cliniche. Le forme ricorrenti hanno una prognosi peggiore rispetto a quelle recidivanti (Tab. II). Le nuove linee guida suggeriscono pertanto di considerare, in questi casi, il timing della rimozione del catetere peritoneale. Le indicazioni alla rimozione del catetere peritoneale rimangono le stesse: peritoniti refrattarie e recidivanti, peritoniti micotiche ed infezioni refrattarie del tunnel e dell'exit-site. La rimozione del catetere peritoneale può inoltre essere presa in considerazione per peritoniti ripetute, micobatteriche e da microrganismi intestinali multipli (8).

\section{Principali tipi di microrganismo e trattamento}

\section{STAFILOCOCCO COAGULASI-NEGATIVO}

Queste forme di peritoniti sono comuni. Lo Stafilococco epidermidis è il microrganismo più diffuso; questo tipo d'infezione è legata alla contaminazione cutanea ed è una forma moderata che risponde rapidamente alla terapia antibiotica, anche se talvolta può recidivare a causa della formazione del biofilm $(18,19)$. In questo caso è raccomandata la sostituzione del catetere peritoneale (8). A causa della frequente meticillino-resistenza di questi ceppi, si consigliano l'utilizzo della vancomicina per almeno due settimane e la sospensione della gentamicina eventualmente iniziata come terapia empirica.

\section{STREPTOCOCCO ED ENTEROCOCCO}

Questi microrganismi sono la causa di peritoniti particolarmente severe. Recenti studi (20) hanno dimostrato che, in generale, le forme streptococciche tendono a rispondere meglio alla terapia antibiotica. La terapia di scelta rimane l'ampicillina, in associazione a un ami- noglicoside per un'azione sinergica. Le forme di enterococco resistenti alla vancomicina e all'ampicillina possono essere trattate con linezolid o quinuprostina/ dalfopristina. La daptomicina è stata utilizzata con successo in due casi (21).

\section{STAFILOCOCCO AUREO}

È la causa di peritoniti severe da contaminazione cutanea 0 , spesso, da infezione del catetere peritoneale. In quest'ultimo caso, vista l'elevata probabilità d'insuccesso terapeutico, è consigliata la rimozione del catetere (8). La terapia prevede la sospensione della gentamicina e la prosecuzione del trattamento con vancomicina per 21 giorni. Sulla base di una review del 2007 (22), si suggerisce l'aggiunta di rifampicina come prevenzione di peritoniti recidivanti o ricorrenti, escludendo interazioni con altre terapie croniche in atto.

\section{CORYNEBACTERIUM}

Nelle nuove linee guida è inserito un paragrafo sulle peritoniti determinate da questo microrganismo. Benché non siano forme di comune riscontro, numerose sono le segnalazioni d'infezioni recidivanti e ricorrenti da corynebacterium (23). In questi casi sembra che un ciclo di 21 giorni di vancomicina sia sufficiente a determinare la guarigione. Nelle forme refrattarie, se la rimozione del catetere avviene entro una settimana, esiste un rischio ridotto di passaggio all'emodialisi.

\section{PSEUDOMONAS AERUGINOSA}

Questa forma di peritonite è spesso correlata allinfezione del catetere, per cui è necessaria la rimozione dello

\section{TABELLA II - TERMINOLOGIA DELLE PERITONITI}

\section{DEFINIZIONI DELLE PERITONITI}

Ricorrente: un episodio che si verifica entro 4 settimane dal completamento della terapia per un precedente episodio causato da un microrganismo differente

Recidivante: un episodio che si verifica entro 4 settimane dal completamento della terapia per un precedente episodio, causato dallo stesso microrganismo, o con coltura negativa

Ripetuta: un episodio che si verifica dopo più di 4 settimane dal completamento della terapia per un precedente episodio causato dallo stesso microrganismo 
stesso. L'andamento è in genere severo: uno studio recente ha dimostrato che le peritoniti da Pseudomonas sono associate a frequenti ospedalizzazioni, alta incidenza di rimozioni del catetere e trasferimento permanente all'emodialisi (24). La terapia prevede l'utilizzo di due antibiotici con differente meccanismo d'azione. Lo schema più usato comprende un fluorochinolonico per os associato a piperacillina (4 g e.v. ogni 12 ore), oppure, in alternativa, a ceftazidime, cefepime o tobramicina.

\section{INFEZIONE DA ALTRO GRAM-NEGATIVO}

Le peritoniti da altro gram-negativo (E. coli, Klebsiella, Proteus) derivano, in genere, dalla contaminazione da contatto, dall'infezione dell'exit-site o dalla migrazione trans-murale in corso di diarrea, diverticolite o colite. L'antibiotico deve essere scelto sulla base dell'antibiogramma (ceftazidime o cefepime i più indicati), sebbene in questi casi il batterio possa frequentemente presentare una sensibilità inferiore in vivo rispetto a quella riscontrata in laboratorio (8). Nel 2006 uno studio retrospettivo ha evidenziato un rischio maggiore di resistenza per quei pazienti sottoposti poco tempo prima ad altra terapia antibiotica (25).

\section{PERITONITI POLIMICROBICHE}

Le infezioni da microrganismi enterici fanno seguito a patologie sottostanti del tratto gastroenterico. Il riscontro in associazione a batteri anaerobi merita una valutazione chirurgica, visto l'elevato rischio di morte (26). Il metronidazolo, in associazione ad ampicillina, ceftazidime o un aminoglicoside resta la terapia di scelta.

Nel caso d'infezioni polimicrobiche da gram-positivi, la prognosi è più favorevole. La mancata risposta alla terapia e l'infezione del catetere costituiscono indicazione alla rimozione (8).

\section{PERITONITI MICOTICHE}

La peritonite micotica rappresenta una seria complicanza che fa seguito a terapie antibiotiche. L'elevato rischio di morte impone, isolato l'agente, la rimozione del catetere peritoneale. La terapia iniziale si avvale dell'associazione tra anfotericina B e flucitosina, sino a quando non è disponibile l'esame colturale. Successivamente un'echinocandina, il fluconazolo, il posaconazolo o il voriconazolo possono sostituire l'anfotericina $\mathrm{B}$, la cui somministrazione intraperitoneale è causa di peritoniti chimiche e l'utilizzo endovena comporta una scarsa disponibilità peritoneale (8). Le echinocandine sono state utilizzate con successo nel trattamento delle peritoniti da Aspergillo e da Candida resistenti o nei pazienti con intolleranze farmacologiche (27). Voriconazolo ( $200 \mathrm{mg}$ e.v. due volte al dì per 5 settimane) e posaconazolo (400 $\mathrm{mg}$ due volte al dì per 6 mesi) sono stati utilizzati con successo per trattare le peritoniti da Mucormicosi resistenti $(28,29)$. La flucitosina richiede il monitoraggio della concentrazione sierica per evitare la tossicità midollare.

\section{Esame colturale negativo}

L'esame colturale può essere negativo per una serie di ragioni tecniche e cliniche (validità delle metodiche, microrganismi rari, uso di antibiotici per altre ragioni). Nella pratica clinica, la maggior parte di queste peritoniti sono determinate da gram-positivi. Se l'inizio della terapia antibiotica empirica determina un miglioramento del quadro clinico, questa va proseguita per due settimane; in caso contrario sarebbe opportuno considerare la rimozione del catetere. In confronto alle forme con esame colturale positivo, esse mostrano una migliore risposta alla terapia antibiotica, e un minor rischio di complicanze (30).

\section{Durata del trattamento}

La terapia antibiotica dovrebbe avere una durata non inferiore alle due settimane. I pazienti che rispondono più lentamente al trattamento, indipendentemente dalla rimozione del catetere o meno, devono proseguire la terapia per almeno tre settimane (8).

\section{Conclusioni}

Le linee guida del 2005, rispetto alle precedenti, riconobbero la mancanza di evidenza della superiorità di un regime antibiotico sugli altri e contribuirono a ridimensionare il problema della resistenza alla vancomicina. Inoltre non diedero indicazioni precise sugli antibiotici da usare, ma consigli circa l'impiego di antibiotici efficaci sui microrganismi più diffusi.

Le nuove linee guida mantengono sostanzialmente la stessa impostazione. Gli studi effettuati negli ultimi cinque anni, in alcuni casi hanno contribuito ad ampliare la scelta degli antibiotici da utilizzare nei casi di resistenza o d'intolleranza, problematiche comunque rilevanti nell'ambito delle malattie infettive, pur non supportando con forte evidenza le opinioni delle precedenti linee guida, né apportando modifiche sostanziali al trattamento delle peritoniti. 


\section{Riassunto}

La peritonite rappresenta la principale complicanza legata alla dialisi peritoneale. Sin dal 1983, l'International Society of Peirtoneal Dialysis (ISPD) ha elaborato delle linee guida, revisionate e aggiornate più volte sino al 2010, su prevenzione, diagnosi e trattamento delle infezioni correlate alla dialisi peritoneale.

Tutti i pazienti in trattamento peritoneo-dialitico, che presentano un liquido di drenaggio torbido, devono iniziare una terapia antibiotica empirica, ad ampio spettro, per via intraperitoneale ed eseguire esami colturali. La prima scelta resta la vancomicina associata a cefalosporine di terza generazione o aminoglicosidi. In seguito la terapia deve essere modificata sulla base dell'antibiogramma. La rimozione del catetere peritoneale è indicata in caso di peritoniti refrattarie e recidivanti, micotiche, infezioni croniche del tunnel e dell'exit-site. La terapia deve avere una durata non inferiore alle due settimane. I pazienti che rispondono lentamente, devono proseguire la terapia per almeno tre settimane.

Le nuove linee guida, come quelle del 2005, non forniscono indicazioni precise sugli antibiotici da usare. Gli studi effettuati negli ultimi cinque anni hanno ampliato le possibilità terapeutiche nei casi di resistenza o intolleranza, pur non supportando con forte evidenza le opinioni presenti nelle precedenti linee guida.

Parole chiave: Linee guida, Dialisi peritoneale, Peritonite, Antibiotici

\section{Indirizzo degli Autori:}

Roberto Russo, MD

UO di Nefrologia

Azienda Ospedaliero Universitaria

Consorziale Policlinico di Bari

Piazza G. Cesare, 11

70124 Bari

roberto.russo@policlinico.ba.it

\section{BIBLIOGRAFIA}

1. Fried LF, Bernardini J, Johnston JR, Piraino B. Peritonitis influences mortality in peritoneal dialysis patients. J Am Soc Nephrol 1996; 7:2176-82.

2. Woodrow G, Turney JH, Brownjohn AM. Technique failure in peritoneal dialysis and its impact on patient survival. Perit Dial Int 1997; 17:360-4.

3. Choi P, Nemati E, Banerjee A, Preston E, Levy J, Brown E. Peritoneal dialysis catheter removal for acute peritonitis: a retrospective analysis of factors associated with catheter removal and prolonged postoperative hospitalization. Am J Kidney Dis 2004; 43:103-11.

4. Bayston R, Andrews M, Rigg K, Shelton A. Recurrent infection and catheter loss in patients on continuous ambulatory peritoneal dialysis. Perit Dial Int 1999; 19:550-5.

5. Bunke CM, Brier ME, Golper TA. Outcomes of single organism peritonitis in peritoneal dialysis: gram-negatives versus gram-positives in the Network 9 Peritonitis Study. Kidney Int 1997; 52:524-9.

6. Johnson DW, Dent H, Hawley CM, et al. Associations of dialysis modality and infectious mortality in incident dialysis patients in Australia and New Zealand. Am J Kidney Dis 2009; 53:290-7.

7. Levy J, Brown E, Daley C, Lawrence A. Peritonite. Il Manuale Oxford - Dialisi, Edizione italiana a cura di Maurizio Gallieni:Edi.Ermes S.r.1., 2010; 342.

8. Li PK, Szeto CC, Piraino B, et al. Peritoneal dialysis-related infections recommendations: 2010 update. Perit Dial Int 2010; 30:393-423.

9. Lui SL,Cheng SW, Ng SY, et al. Cefazolin plus netilmicin versus cefazolin plus ceftazidime for treating CAPD peritonitis: effect on residual renal function. Kidney Int 2005; 68:2375-80.

10. Goffin E, Herbiet L, Pouthier D, et al. Vancomycin and ciprofloxacin: systemic antibiotic administration for peritoneal dialysis-associated peritonitis. Perit Dial Int 2004; 24:433-9.

11. Lima RC, Barreira A, Cardoso FL, Lima MH, Leite M Jr. Ciprofloxacin and cefazolin as a combination for empirical initial therapy of peritoneal dialysis-related peritonitis: five-year follow-up. Perit Dial Int 2007; 27:56-60.

12. Kobayashi K, Nakamoto H, Okada S, et al. Efficacy and safety of meropenem plus tobramycin followed by meropenem plus vancomycin for treating peritonitis in patients on continuous ambulatory peritoneal dialysis. Adv Perit Dial 2006; 22:65-8.

13. Zelenitsky S, Barns L, Findaly I, et al. Analysis of microbiological trends in peritoneal dialysis-related peritonitis from 1991 to 1998. Am J Kidney Dis 2000; 36:1009-13.

14. Blunden M, Zeitilin D, Ashman N, Fan SL. Single UK centre experience on the treatment of PD peritonitis-antibi- 
otic levels and outcomes. Nephrol Dial Transplant 2007; 22:1714-19.

15. Chow KM, Szeto CC, Cheung KK, et al. Predictive value of dialysate cell counts in peritonitis complicating peritoneal dialysis. Clin J Am Soc Nephrol 2006; 1:768-73.

16. Szeto CC, Chow KM, Wong TY, et al. Feasibility of resuming peritoneal dialysis after severe peritonitis and Tenckhoff catheter removal. J Am Soc Nephrol 2002; 13:1040-5.

17. Szeto CC, Kwan BC, Chow KM, et al. Recurrent and relapsing peritonitis: causative organisms and response to treatment. Am J Kidney Dis 2009; 54:702-10.

18. Finkelstein ES, Jekel J, Troidle L, Gorban-Brennan N, Finkelstein FO, Bia FJ. Patterns of infection in patients maintained on long-term peritoneal dialysis therapy with multiple episodes of peritonitis. Am J Kidney Dis 2002; 39:1278-86.

19. Dasgupta MK, Ward K, Noble PA, Larabie M, Costerton JW. Development of bacterial biofilms on Silastic catheter materials in peritoneal dialysis fluid. Am J Kidney Dis 1994; 23:709-16.

20. O'Shea S, Hawley CM, Mc Donald SP, et al. Streptococcal peritonitis in Australian peritoneal dialysis patients: predictors, treatment and outcomes in 287 cases. BMC Nephrol 2009; 10:19.

21. Huen SC, Hall I, Topal J, Mahnensmith RL, Brewster UC, Abu-Alfa AK. Successful use of intraperitoneal daptomycin in the treatment of vancomycin-resistant enterococcus peritonitis. Am J Kidney Dis 2000; 36:1183-92.

22. Szeto CC, Chow KM, Kwan BC, et al. Staphylococcus aureus peritonitis complicates peritoneal dialysis: review of 245 consecutive cases. Clin J Am Soc Nephrol 2007; 2:245-51.
23. Barraclough K, Hawley CM, McDonald SP, et al. Corynebacterium peritonitis in Australian peritoneal dialysis patients: predictors, treatment and outcomes in 82 cases. Nephrol Dial Transplant 2009; 24:3834-9.

24. Siva B, Hawley CM, McDonald SP, et al. Psudomonas peritonitis in Australia: predictors, treatment, and outcomes in 191 cases. Clin J Am Soc Nephrol 2009; 4:957-64.

25. Szeto CC, Chow VC, Chow KM, et al. Enterobacteriaceae peritonitis complicating peritoneal dialysis: a review of 210 consecutive cases. Kidney Int 2006; 69:1245-52.

26. Faber MD, Yee J. Diagnosis and management of enteric disease and abdominal catastrophe in peritoneal dialysis patients with peritonitis. Adv Chronic Kidney Dis 2006; 13:271-9.

27. Matuszkiewicz-Rowinska J. Update on fungal peritonitis and its treatment. Perit Dial Int 2009; 29 (Suppl 2):S161-5.

28. Ghebremedhin B, Bluemel A, Neumann KH, Koenig B, Koenig W. Peritonitis due to Neosartorya pseudofischeri in an elderly patient undergoing peritoneal dialysissuccessfully treated with voriconazole. J Med Microbiol 2009; 58:678-82.

29. Sedlacek M, Cotter JG, Suriawinata AA, et al. Mucormycosis peritonitis: more than 2 years of disease-free followup after posaconazole salvage therapy after failure of liposomal amphotericin B. Am J Kidney Dis 2008; 51:302-6.

30. Fahim M, Hawley CM, McDonald SP, et al. Culture-negative peritonitis in peritoneal dialysis patients in Australia: predictors, treatment and outcomes in 435 cases. Am J Kidney Dis 2010; 55:690-7. 